\title{
Threshold concepts and certainty: a critical analysis of 'troublesomeness'
}

\author{
Richard Stopford ${ }^{1}$ [D
}

Accepted: 14 September 2020 / Published online: 3 October 2020

(c) The Author(s) 2020

\begin{abstract}
The threshold concept framework is a key contemporary theory in pedagogy. The core idea is that 'threshold concepts' are distinctively 'troublesome' for students and act as gatekeepers to their disciplines. No doubt the theory is compelling because there is surely something right about this. Student difficulty with conceptual material is familiar to all teaching practitioners. Furthermore, to avoid rote levels of understanding, mastery of discipline-specific conceptual material is key. However, TCF has struggled to articulate key dimensions of its theory: it is without a methodology for identifying threshold concepts. It has also faltered in explaining how student difficulty is a function of difficulties endemic to the concepts, rather than as a contingent phenomenon about individual students.

I offer a novel way to think about identifying threshold concepts, and for theorising student difficulties which may arise from those concepts. I argue that there is an 'existential' kind of certainty which acts as a framework within which epistemic activities take place. Disciplines which theorise concepts in ways that clash with students' existential certainties are candidates for threshold concepts and may generate 'objective' difficulties for students. As much as I think theorising existential certainty helps TCF overcome theoretical challenges, it would require revisions to the way that it is currently being theorised and applied. I also believe that even without attachments to TCF, 'existential certainty' is a real phenomenon, shaping the very possibilities of student experience, and which any pedagogical theory should be aware of.
\end{abstract}

Keywords Threshold concepts · Wittgenstein · Certainty · Rowbottom · Epistemology · Gender theory

\section{Preliminaries}

In 2003, Land and Meyer posed an innovative way for thinking about concepts within disciplines and the troubles they pose in student learning. Whilst the threshold concept framework (TCF) is theoretically complex, the idea is straightforward and has potential import for any teaching practice. A significant source of student learning difficulty is

Richard Stopford

r.j.stopford@durham.ac.uk

1 Durham University, Durham, UK 
due to discipline-specific concepts that must be mastered in order for students to successfully participate in the discipline. For example, if a student does not understand 'gravity', how are they going to study physics given that gravity plays such an important role in a great deal of physics' theory and practice? 'Gravity' is preponderant throughout physics' theories and practice; our conception of that phenomenon shapes and inflects the rest of the conceptual apparatus of physics' theories. A student must do more than learn some discrete packet of information codified within a concept-no matter how important. A deep understanding of 'gravity' requires understand the complex ways that 'gravity' suffuses physics theories and practice. Until a student has this deeper understanding of this concept-amongst others of course-they will find themselves on the outside of the discipline looking in. By contrast, to have deep understanding of the concept is to step over the threshold/boundaries of the discipline into an insider position, as it were.

If Land and Meyer are right about threshold concepts (TC) as crucial for deep learning, this has immediate general effects for practitioners in higher education, and teachers more generally. Firstly, these concepts must be identified by teachers as (conceptual/theoretical) gatekeepers of the discipline. As such, the TCF must be able to help practitioners to disentangle and accommodate for, special difficulties in students' learning-after all, no one denies that students often find concepts hard to grasp. Finally, given the supposed scope of influence of TCs throughout disciplines, practitioners must be aware of the affective effects on the students: to not grasp a threshold concept is to implicate a student's relationship to the discipline and all the frustrations and stress which attend that. In short, threshold concepts are a pedagogical framework for thinking about learning experience, coordinated around student difficulties and transformations which revolve around special kinds of concepts.

If the threshold concept framework (TCF) properly theorises learning structures and distinctions in student difficulty and is an apt model for coordinating teaching practice in the face of such difficulties, it demands our attention. And it does have our attention. This is unsurprising given that any teacher and pedagogue knows the irreducibility of student difficulty; student troubles are everyone's problems. Since 2003, TCF has generated a huge amount of theoretical and empirical interest. Yet, TCF is under an unusual degree of theoretical pressure on three counts. Firstly, because it is a pedagogical framework coordinated about concepts, and it inherits all the problems of dealing with such difficult and divisive entities. Moreover, TCF is committed to saying that it has identified, in effect, a new and important class of concepts which then needs theorising, establishing and justifying. Third, TCF does more than draw our attention to the idea that disciplines have challenging, bespoke concepts - an important yet somewhat uncontroversial point. Rather, TCF makes the stronger claim that what makes these concepts distinctive is that they are inherently difficult to learn. Indeed, TCF makes the strong claim that TCs just are troublesome to learn, and that this troublesomeness is indexed to the role and situation in the discipline. So, on the one hand, TCF offers us an entire framework for thinking through pedagogical theory and practice, but it can only do so on the basis that it makes good on these points of theory. If it cannot do so, it may be that, at best, it reminds us that concepts can often be tricky to understand. This is clearly important but hardly a pedagogical framework for theory and practice. I argue that, in its current form, it does no more than this. Nevertheless, I want to argue that with some significant revisions in how student difficulty is theorised, it can do much more: it can identify a distinctive class of concepts; these concepts will pose difficulties for some students for more fundamental reasons than they are just tough to learn. This, I take it, is a form of TCF that does demand our attention. In so doing, I aim to make some 
important distinctions about student difficulties in their learning experience which will be relevant even if the reader is not convinced by, or attached to, TCF.

\section{Locating 'certainty' in threshold concepts}

It is clear that TCF must establish its theoretical commitments before we can take it seriously as model for practice. Why look for threshold concepts if we cannot establish that there are such concepts? Why think that student difficulty is something about the concepts rather than something about individual student experience? Arguably, TCF has done poorly in addressing both theoretical challenges. What a TC is, what its essential features are, and how we identify them-if there even are such concepts-remains unresolved. Originally Meyer and Land (2003: 4-5; 2005) argued that TCs were 'transformative' of student experience, 'liminal' insofar as their acquisition involves a period of disturbance as a learner, 'irreversible' in that once understood they cannot be unlearned as it were, determining of disciplinary 'boundaries', and intrinsically 'troublesome' to learn. Land has since extended the criteria (2011). Furthermore, the criteria themselves have been contested (Male and Baillie 2011; Foley (2011); . These contestations have been surveyed and explored by Quinlan et al. (2013: 586-7) and Barradell (2013: 266-9). It is not necessary, here, to adjudicate between these competing conceptions of the view: I will focus on one trajectory for developing TCF revolving around the characteristic 'troublesomeness' of certain concepts (Taylor 2006: 90-1; 2008: 186; Cartensen and Bernhard 2008: 144). That is not to suggest that the necessity and sufficiency of troublesomeness have been uncontested (Hill, 2020: 673-5). Nevertheless, I think the following analysis goes some way to addressing Hill's concerns and making a case for the value of troublesomeness as defining of TCs. How then do we think about 'troublesomeness' as the core of the theory? One way is through TCs' affects: difficult concepts will have an impact on students' emotional wellbeing (see Rattray, 2016: 71-2; Timmermans and Meyer, 2019: 51-67). Not wanting to be too schematic by pulling apart the affective from the cognitive, but what of the cognitive dimension? In the literature, there is a move to thinking about the cognitive dimension of troublesomeness in terms of 'uncertainty' (Land, 2016: 11).

What, then, is 'uncertainty'? The purpose of this paper is to explore the phenomenon of 'uncertainty' and how it might help clarify the troublesome nature of threshold concepts: what they are, and how they are problematic for students. Land rightly highlights the way that uncertainty can be a good thing in student experience: it can be a form of openness and flexibility in student understanding. Nevertheless, this point does not help clarify the theoretical foundations of threshold concepts. Nor does it imply any special relationship between uncertainty and TCs. That said, I think that there is something promising about this idea, albeit with a shift of emphasis. It is not uncertainty, but 'certainty' that could really help finesse the theoretical foundations of TCF. I want to explore the idea that certainty is the better index of student 'troublesomeness' because it is when student certainties clash with discipline-specific conceptual content that some of the most profound difficulties in students' learning experience arise. Furthermore, this is not just any kind of certainty, but a distinctive, fundamental level of certainty that I will be referring to as 'existential certainty'-inspired by the work of the philosopher, Wittgenstein. What I mean by this will become clearer through the course of the paper. For now, think of 'existential' as whatever pertains the meaningfulness of life as such. Existential certainties are the sureties out of which a meaningful life is constituted and which make possible a solid foundation 
for particular kinds of meaningfulness-semantic, epistemic, normative. If TCF can analyses 'troublesomeness' by way of 'existential certainty', and this is at the foundational level of student experience, we may have found a good way to theorise the theoretical foundations of TCF, its special concepts, and the distinctive kinds of difficulty that attends those concepts.

Indeed, it is crucial that TCF is able to give a theory of its own concepts, and of the distinctiveness of the difficulty that attends those concepts because, as already mentioned, TCF has been strongly challenged on both of those counts. Rowbottom (2007: 268) argues that TCF does not have a working account of how to identify threshold concepts, or that the difficulty of those concepts resides in the concept itself, rather than in the particularities of student abilities and experience. These challenges have sometimes been noted but never fully tackled in subsequent literature (see Nicola-Richmond et al., 2018). First, he has argued that TCF is unable to provide any clear theory for how we identify TCs (See also O'Donnell, 2010: 2). None of the characteristic features of TCs offered in primary literature - transformation, liminality, irreversibility and boundedness-are, by their own admission necessary or sufficient. As such we have very little solid ground for saying some concept, 'gravity' say, is a threshold concept of physics, but 'heat' is not. Assume 'gravity' has all the characteristics but 'heat' only one. Is 'gravity' a TC, but not 'heat'? We cannot tell because the progenitors of the theory admit that sometimes a concept has all the characteristics and sometimes not. It is just too loose a theory as it stands. TCF must provide some kind of in principle way to define and thereby provide identity conditions for threshold concepts. Salwén has gone on to analyse a host of possible semantic responses to deal with the definitional problem, concluding that none are successful (2019: 2-8); I agree. In fact, TC theorists still need to convince us that there are such concepts! Current empirical work is dangerously close to being both a 'shot in the dark' and methodologically circular: given that the identity conditions of threshold concepts are deeply contested, how can we use the theory to confidently identify them? More to the point, we had better not be using an already too elastic theory to identify threshold concepts, then using those 'threshold concepts' as evidence for the theory. Indeed, I worry that this may be happening given the methodological outline in studies such as that of White et al. (2016: 55-6)—see also Salwén, (2019: 9-12).

Rowbottom's second argument is just as damaging. TCF claims that TCs are intrinsically difficult to acquire. However, as he argues with the 'gravity' example, anecdotal evidence of student learning clearly shows that some students will find a concept difficult and some will not. Difficulty is not endemic to the concept. Concepts are not intrinsically difficult, rather they are difficult for a student given the student's experience and capabilities. Student difficulty has to be treated as phenomenon about students, not about concepts as such (2007: 268). If he is right-and there is surely something right about the idea that difficulty is a function of individual student situations - this is a significant blow for TCF given its claims that TCs are (intrinsically) difficult. Indeed, it is only due to this supposed intrinsic difficulty that TCF theorises 'threshold concepts' as disciplinary phenomena. Land and Meyer try to coordinate this difficulty in their paper, 2005: 380; and, in Meyer et al. (2008). However, I am sceptical of their success largely because they are working with an intrinsic - read: necessary-notion of conceptual difficulty on the one hand, and trying to square that with contingent student experience on the other. This modal clashbetween necessity and contingency — cannot be theorised away by clarifying structural stages in learning. It is not the contingency that Row bottom, or I, dispute, but that the putative necessity of conceptual difficulty is consistent with the actual contingencies of student experience. This does not make modal sense. 
Clearly the problems get somewhat abstract, and philosophical. However, this is the ground that TCF finds itself in given its claims. Moreover, these are not just abstract skirmishes in theory. To the extent that TCF contributes to a general understanding of student difficulty — which no one doubts is a real phenomenon - then failing to establish theoretical grounds for analysing and practically responding to student difficulties is a missed opportunity. I mobilise my analysis of certainty by way of these two problems precisely because TCF is under considerable theoretical pressure, and because the clarification that 'existential certainty' could bring to TCF actually helps to dissolve both of these core issues. I think we can use the effects of students' existential certainties in the classroom as a means for identifying TCs. This could solve Rowbottom's first objection. More importantly perhaps, existential certainty will help ground student difficulty in something beyond the contingent situation of individuals. I will argue that existential certainties are effectively expressions of socio-cultural states of affairs. When I am existentially certain of something, we could say that I am expressing how the world is for me. Yet, such certainties are not so much my own acquired beliefs, as a foundational framework of intelligibility upon which I can build beliefs. So, existential certainties are articulations of fundamental attachments about how the world is; they are functions of enculturation, of being a member of a culture. As such, all of us take on various certainties in a way that constitutes our being part of a culture and which we share with others who are also members of that culture. So, if certainties are not really products of individuals' experiences but the cultural frameworks through which students make sense of themselves and the world around them, then we can locate a distinctive modality of student difficulty: I may express what seems like a personal difficulty, but when the source of that difficulty is a clash of disciplinary material with what I am certain of, the trouble is not merely subjective, but indicative of something at the socio-cultural level. In such cases we have a basis for thinking that some discipline specific conceptual material will be 'objectively' troubling for some constituencies of students given their sociocultural background. Furthermore, such objective troublesomeness would be consistent with empirical evidence that the 'troublesomeness' of any candidate threshold concept is only experienced contingently by some students. Being able to clarify the identity conditions for threshold concepts, and being able to assert clear, 'objective conditions' for the 'troublesomeness' of some discipline specific material goes a long way to consolidating TCF as a robust theory of a distinctive class of concepts.

Nevertheless, my argument comes at a price for the theory. Such existential certainties are readily identifiable, but that is because they occupy a distinctive role for epistemic agency: certainties constitute the framework which makes possible any epistemic activities such as learning, knowing, thinking, believing, reasoning, etc. As such, certainties are, properly speaking, prior to knowledge. This would be a problem for TCF as it currently stands because most examples of threshold concepts in the TCF literature, and the way that attendant student difficulties are characterised, are epistemic, i.e. pertaining to knowledge. I cannot hope to survey all examples of candidate concepts here, but see for example: 'proportional reasoning' and 'opportunity cost' in economics (Lloyd, \& Frith, 2013; Shanahan, 2016 respectively), 'representation' in philosophy (Booth, 2006); 'osmosis' in biology (Taylor, 2006), etc. So, much that is taken in the literature as a candidate for a threshold concept because of problems of understanding, no matter how 'deep', would not count as certainty-involving in the relevant way. If I am right, then either TCF has to bite the bullet that certainties constitute TCs, but then TCs are not epistemic material in the way they have been currently theorised, or reject my view of 'existential certainty' and lose its advantages in identifying the TCs and grounding 'objective troublesomeness'. 
The second cost of my analysis is that the difficulties associated with TCs by way of existential certainty are not intrinsic to TCs vis-a-vis their role in a discipline. Difficulty is, as Rowbottom points out, a relational state of affairs. But he sees this as a relation simply between the student and the discipline. Again, this would make all student difficulty contingent on the predicaments of individual students. This is important in its own right, but a deviation away from the claims of TCF. Existential certainty allows us to think about the relation as a clash between socio-cultural forms of living on the one hand which constitute the epistemic frameworks within which particular students operate, and discipline-specific material on the other. The problem is that, whilst this means that disciplinary boundaries may help to coordinate us towards 'troublesome' material, student difficulties indexed to existential certainties are not simply functions of disciplinary features, i.e. of concepts and the difficulty that inheres in them due to their role in a discipline. So, we cannot look inwards to disciplines to theorise TCs; rather, we must look at the complex mediations of individual student experience, socio-cultural background, alongside disciplinary content. I say this is a cost, but only to the theory as it currently stands. I would argue that my analysis simply focuses our attention on the socio-cultural context within which disciplines are situated, rather than simply at disciplines, and that is as it should be.

Land has already made the link between threshold theories and uncertainty. This is a good place to begin: tracing some existing links in the literature. He thinks about the way that uncertainty is endemic to learning. The fact that TCF's account of troublesomeness acknowledges and finds a pedagogical place for uncertainty is a virtue of the theory. This is fine as far as it goes. However, he does not provide an analysis of uncertainty, nor could his use of the notion meet the concerns raised above. This is not a problem given he is not trying to address these issues. Nevertheless, I will consider some possible ways that we could think about certainty to do just that, and then explicating what is so distinctive about this view. I will then characterise the relevant modality of certainty as existential, arguing that it can be deployed to meet the challenges for TCF raised above. But also, how it would require us to think differently about TCF if it is adopted. I will finish by considering an example from my own teaching in philosophy, demonstrating a situation of 'objective troublesomeness'.

\section{Preparing students to be uncertain in an uncertain world: disentangling 'epistemic confidence' from 'certainty'}

I want to consider Land's engagement with the notion of 'certainty'. In his paper Toil and Trouble: Threshold Concepts as a Pedagogy of Uncertainty (2016), Land argues that 'uncertainty' is both irreducible in a progressive educational experience (2016: 16 and 20), and that it provides a bulwark against the commodification of education that requires a kind of commodified 'certainty' (2016: 17). For the purposes of this paper, I want to set aside the political point—no matter how sympathetic I am-and focus on the epistemic dimensions of the argument. In a little more detail, I take it that he is arguing for two points (1 and 2) that have epistemic import, and a normative point (3) about the value of TCF given these epistemic points (2016: 21).

1 The world is a complex and dynamic place. We cannot future-proof disciplines with fixed ontological material when novel states of affairs are constantly emerging. Good theorising must be able to be responsive to future changes, and some appropriate need 
for ontological pluralism. (2016: 16 - 7) Moreover-a point emphasised by way of Shulman-the complexity of the 'real world' is such that the theories we teach students do not perfectly, and without controversy, explain/predict/analyse the world we are teaching them to understand. To put it another way, theories are underdetermined in their realworld applications, answers and solutions. As such, peddling an educational 'product' that is reified to the point of 'certainty' will thereby falsify the very phenomena which theories seek to grip (Land, 2016: 11, quoting Shulman, 2005: 1).

2 A corollary point of (1) is that being 'certain' as a student is to have a fixed way of thinking that is inappropriate for understanding and explaining dynamic, complex real-world phenomena (2016: 20 - 2).

3 TCF theorises 'troublesomeness' and 'transformation' as endemic to both learning and teaching, and is therefore appropriate to coordinating the uncertainties engendered in 1 \& 2 (2016: 18 - 20) —of course Land, quite reasonably, wants to make a further value claim for TCF given the political points I have sidelined here.

I am sympathetic to all these points. Focusing on (1) and (2) for now, the idea that theories cannot provide students with complete certainty-and should not hope to-is surely right. The question is, 'right' how? To motivate his paper, Land points to Shulman's Pedagogy of Uncertainty (2005) and the latter's reflections on medical pedagogy. Understanding Shulman's view of 'uncertainty' might help us to analyse the uncertainty involved in (1) and (2). Shulman's point, as I understand it, is that when medical practitioners apply their medical training in real-world scenarios there is always the possibility that they could get it wrong. The real world (of disease and pathology) is so complex that it is usually the case that some symptom/pathology is consistent with a different diagnosis/treatment. Medical practitioners have to make important judgments on the basis of theory, and learnt practice, given that both tend to underdetermine the case at hand. Still, despite this underdetermination, they must be able to tell the difference between a good judgement and a bad judgement. And they have to live with the consequences (Shulman, 2005: 19-20).

Land and Shulman both think about such situations, quite reasonably, in terms of 'certainty' and 'uncertainty'. They do not analyse these terms; however, I think they have something like the following in mind. The doctor diagnoses her patient as best she can, given appropriate understanding of theory and acquired evidence. The patient asks her, 'Is she certain that he has Lupus?' She responds, 'No. But it is my medical opinion that it is'. One thing that medical students need to be taught is what constitutes a good medical opinion-presumably some complex admixture of theory, practice, intuition, along with sound, relevant, common sense. Medical students must also be taught how to live with this 'uncertainty'. For medical practitioners, some degree of 'certainty' is appropriate, but hubris is not. Nevertheless, decisions must be made whilst being 'uncertain' because rampant 'uncertainty' may be as dangerous as hubris.

If I have teased out the relevance of 'uncertainty' correctly in this example, what seems to be happening here is that medical students have to assess their judgements. The doctor judges that the patient has $x$. But the doctor must also assess that judgement itself alongside other possibilities, contingencies, risk management, etc. In other words, there is a 'first-order' epistemic act: the diagnosis, $p$, that ' $x$ has $y$ '. And there is a 'second-order' epistemic act which is the evaluation of that first-order judgement: I judge that ' $p$ ' (' $p$ ' being the diagnosis) is a good judgement. The two orders are distinguishable insofar as they are based on different categories of material. The first-order 
judgement is directly concerned with empirical states of affairs, e.g. symptoms. The second-order judgement is concerned with the practice and theory choice involved in the diagnostic management of the empirical evidence. We might express the secondorder judgement in terms of a propositional attitude: I am confident (propositional attitude indicating second-order judgement) that $x$ has $y$ (first-order judgement). In this scenario the concept of uncertainty seems to be a way of talking about the tolerability of epistemic confidence in the first order judgement. The doctor might not be certain, but complete certainty is the wrong bar of epistemic confidence given her knowledge, understanding, evidence, and given the nature of diagnosis. A crucial part of her education is learning what the appropriate degree of epistemic confidence in her diagnosis ought to be.

What has this to do with theorising TCF? In the details, not very much. Land does not say how it is that he was inspired by Shulman, and what about the medical situation explored in that paper is directly relevant to theorising TCF. He gestures towards a broad lesson, however: it's okay to be uncertain. To that extent, TCF converges with the example. TCF students come across difficult conceptual material, and experience some form of epistemic uncertainty. In the case of the medical student we can see that this is simply an appropriate part of the practice. As Shulman also suggests, this could be part of a general lesson for students (2005: 22): it's okay to find yourselves 'uncertain'. Indeed, some degree of uncertainty can be an index of good practice and understanding!

However, I think we need to be aware of some slippage here. The TCF claim concerns understanding and assimilation of discipline-specific conceptual material. In the medical student case, it may be that they have understood the concepts and practices of medical theory very well, and had no difficulty assimilating that material. This is because the issue of 'uncertainty' being explored above is a second-order issue of confidence in their use and application of some medical theory, not a first-order uncertainty expressing the status of their understanding of the theory/concept as such. So, we need to be careful taking 'uncertainty' in one epistemic register as a guide for theorising about uncertainty in another register. More to the point, presumably someone could still see the role of second-order uncertainty, without any commitment to uncertainty/ troublesomeness being endemic to discipline-specific conceptual material. In other words, we do not need TCF and there might be other more useful pedagogical theories for thinking through the example. Indeed, complexity theory as a modality of activity theory may be such an alternative. See Bleakley and Cleland's (2015) analysis of medical practice involving non-linear problems and 'emergent properties' of complexity (83-85) as a way to explain the difficulties arising for the medical student.

Again, this is not the place to adjudicate theory choice. The point is not much follows about TCF from the example. To the extent that this example is a generalisable lesson about teaching and learning, it might sway us towards TCF because this pedagogy is already concerned with student difficulty. Yet, for the reasons just suggested, it is not a strong connection. Whatever association TCF has with second-order uncertainty, it does not seem to help us with either of the problems raised in part one: identifying TCs, and establishing an objective ground for student difficulty. The uncertainty the medical student experiences does not pertain to her entry into the discipline but the complexity of her practice having fully entered it. Furthermore, the generality of the difficulties of 'uncertainty' do not pertain to the assimilation and understanding of particular concepts and practice. Rather, how general theories and practice underdetermine the particular complexities of real world cases; the medical example is an issue of application and 
practice. In short, if this is all we had to say about 'uncertainty', I do not think it can do much work either theorising TCF, nor even as a normative reason for adopting TCF.

The example is useful, however, for generating some theoretical distinctions in the epistemic status of 'uncertainty'. Second-order epistemic uncertainty-articulated in terms of confidence as explored in the medical example above-is not the only way to think about the term. I now want to extend the notion of uncertainty in its existential reach. Note that nothing in this additional account of uncertainty undermines the discussion above. What it does do, however, is allow us to think about 'uncertainty' as a fundamental difficulty experienced when students encounter disciplinary conceptual content that clashes with their pre-theoretical 'certainties'. Indeed, it may be better to think of the clashes in disciplinary material with student certainty as an existential disturbance rather than mere uncertainty.

\section{Existential certainty: epistemic frameworks and forms of living}

In the above example, it is not that the medical student is uncertain what a 'diagnosis' is, means, or even what the practice of 'diagnosis' is, but whether or not some diagnosis is the right/best/most appropriate diagnosis; it is a second-order difficulty about practice. In this example, 'certain' signifies very confident; 'uncertain' is not very confident. Perhaps one thinks that this must be the only way to think about these notions. After all, if we are sincere in our use of the term, how often are we ever completely certain? Surely we could always be wrong. In this way, 'uncertainty' and 'certainty' are germane for judging incremental levels of doubtfulness: measurements of degrees of epistemic confidence, tracking cognitive and affective jeopardies in our knowledge-making practices. In this light, 'certainty' and 'uncertainty' exist on a spectrum, delimiting the bounds of epistemic confidence/doubt respectively. Moreover, in such contexts, 'uncertainty' may be a good thing, as it were. I am happy to concede that this is one way we use the two concepts. But against the idea that this is the only way, I want to think through a notion of Wittgensteinian uncertainty.

Before proceeding, some preliminaries about the argument and Wittgenstein's role in the argument are appropriate. The account of certainty and uncertainty developed below is inspired by Wittgenstein. To help readers unfamiliar with his philosophy, I will provide quotations to give some idea of the source. However, a couple of things to note: Wittgenstein's aphoristic style is notoriously gnomic and open to interpretation. As such, Wittgenstein scholarship is often divided. Nothing in the argument of this paper rests on a scholarly fidelity to Wittgenstein. The ideas here are inspired by what I take to be his view. Nevertheless, whether I am right or wrong about Wittgenstein, I hope my account of 'existential certainty' resonates with the reader's own experience, and seems plausible on its own terms. The argumentative issue here is not about Wittgenstein and whether or not he is right, or that I am right about him, but whether or not the account of existential certainty offered here resonates, and helps us to understand the complexities of student difficulty as we find them in the classroom.

Wittgenstein argues that there is a much more fundamental way of thinking about 'certainty' at least: in this fundamental register, certainties are articulations of forms of living which make possible knowledge-making practices (1972: §§94-5). I want to call this kind of certainty 'existential', as opposed to 'epistemic' for reasons which will become clear. This kind of fundamental certainty does not come in degrees, and it does not track confidence in what we know. It also does not exist on a spectrum alongside degrees of 
uncertainty. When one is certain in this way, the thought of being able to be uncertain is unintelligible or absurd. Such certainty is the condition for the possibility of any kind of knowing. With this view of 'certainty' we are all certain of many things, and all of the time. I will now explore this view, distinctions about knowledge-making practices that might arise from it, and how this view of certainty enables us to meet the theoretical challenges to TCF laid out in part one.

Imagine I claim the following: 'It is raining outside'. And someone asks me: 'Are you certain?' I might respond that I am. Only 5 min ago, I looked out of the window and it was raining heavily. The rain was beating on the roof, and it is still doing so. Even though I am not now looking out of the window, I still (strongly) believe it to be raining. But the person I am speaking to tells me I am wrong regardless. I look out the window, exasperated, and to my surprise it is now sunny and the 'rain sound' I can hear is run-off water from a gutter. I'm wrong. When I said I was 'certain', I was expressing a high degree of confidence in my knowledge. As a result of my 'certainty' I was even a touch irritated when someone checked. But it turns out I am wrong; I understand why, and I am prepared to accept that I am wrong. Contrast this very mundane situation with the following. Someone asks me my name; I tell them. But then they want to see some ID. In some scenarios this request is perfectly intelligible: I am trying to board a plane, for example. But imagine that this is nothing to do with my telling the truth, rather they want to check whether or not I know my name-Wittgenstein explores such examples (\$82). This considerably cuts down scenarios in which this request for justification makes sense. Perhaps I have hit my head and the person is a policewoman wanting to check if I'm compos mentis. Now imagine it is just an everyday situation and someone simply wants to know if I know my name-as if I could be mistaken in the same way as I was in the raining example. In such a scenario, I would not give them my ID, or reasons, or evidence. I would tell them that of course I know what my name is, and by telling them my name, I am telling them what is the case, i.e. of how things are beyond the possibility of discussion.

This is not a situation in which I'm prepared to admit of investigation, questioning, incredulity, or any other standard epistemic attitudes or practices. This is because it does not even make sense to me that I might be wrong; I am certain. Indeed, that certainty is so strong, and of a kind, that I am baffled by my interlocutor: that they seem to take it as a live possibility that I might be wrong. This idea that there are times when we are communicating beyond the bounds of standard epistemic practices, e.g. justification, is captured by Wittgenstein in the following, somewhat gnomic aphorisms: To be sure there is justification; but justification comes to an end (\$192). 'It is so difficult to find the beginning. Or, better: it is difficult to begin at the beginning. And not try to go further back’ (\$471). In other words, there are cases in which we have arrived a kind of foundation in which we claim that something simply 'is the case' and there is nothing more to be said: 'Instead of "I know it", one may say in some cases "That's how it is-I rely upon it"” (§176).

What we have in the name example is a very important idea for theorising a fundamental distinction in the structure of epistemology as such, no matter how seemingly trivial the scenario. This situation reveals to us that there are some claims that might appear to be standard, first-order, empirical knowledge claims ("My name is " $x$ "), and so subject to standard epistemic activities such as the giving and taking of reasons, yet are nothing of the sort. Whereas standard 'knowledge' claims admit of doubt, investigation, justification, and evaluation, a genuine certainty precludes such activities; it is not a context where such activities make sense. Indeed, Wittgenstein argues that epistemic activities such as doubting only operate successfully when there is some material that is held fixed. For example, how can you doubt if your doubt is so global that it implicates the language that expresses 
the doubt: 'If you tried to doubt everything you would not get as far as doubting anything. The game of doubting itself presupposes certainty' ( $\$ 155)$. It seems such certainties are not knowledge claims at all precisely because they are not acquired, contestable, and subject to inquiry in the way that knowledge essentially is: 'But I did not get my picture of the world by satisfying myself of its correctness; nor do I have it because I am satisfied of its correctness. No: it is the inherited background against which I distinguish between true and false' (§94). In response to some kind of epistemic incredulity about my name, I am likely to question the intelligibility of our conversation: 'What do you mean, "Are you certain your name is “ $x$ ”?" Of course I am certain!' In short, I do not counter with evidence, reasons, or arguments; I counter by questioning the conditions of intelligibility of the conversation itself. We are not contesting some knowledge, we are already in some disagreement about what counts as appropriate material for conducting epistemic activity.

My entitlement to my name, as something more fundamental than knowledge, is something to do with my very socialisation. That the socio-cultural world is intelligible to me, and that I am intelligible to myself within that socio-cultural space is in part expressed by the naming practices which contribute to situating that entitlement. In such cases I can say that I am indeed completely 'certain'. How the world is for me, and how-I-am-in-the-world is such that I am entitled to claim with certainty that my name is ' $x$ '. Moreover, this is not just something about my comportment in the world, but how I am with others: it is a sociocultural state of affairs. "Now I would like to regard this certainty, not as something akin to hastiness or superficiality, but as a form of life" (\$358). What is certain for me are those shared social practices that congeal as cultures-or 'forms of life'-and which enable me to make sense of myself with others. It is in light of the fundamentality of the certainty involved that I call this certainty 'existential' rather than 'epistemic'. Existential certainties are a condition of my being able to make sense of myself and the world around me; they underwrite meaningfulness as such. As a consequence, uncertainty is not really available in such cases; existential 'certainty' is exhaustive and does not exist on a spectrum with 'uncertainty' ( $\$ 114-5)$.

What this discussion does is bifurcate epistemology, and our epistemic practices, into actual knowledge, and the conditions for the possibility of that epistemic agency. The effects of this for education in general, not just TCF, are significant. On the basis of what has been said so far, we can schematise the analysis of 'certainty' and 'uncertainty' and its relationship to knowledge in the following ways:

\section{Epistemic}

1 'Certainty' and 'uncertainty' as complementary synonyms for 'knowing' and 'not knowing', respectively: I am uncertain that ' $x$ ' may be substituted for I do not know that ' $x$ ';

2 'Certainty' and 'uncertainty' as complementary second-order epistemic attitudes: I am not certain that I know ' $x$ ', but I take it that I do know that ' $x$ ';

3 'Certainty' and 'uncertainty' as complementary phenomenological states involved in one's 'knowledge' of ' $x$ '. I am not certain that I know ' $x$ ' is to reflect on what 'knowing' is like in some instance;

\section{Existential}

4 'Certainty' as a fundamental expression of 'how things are' for the subject: I am certain that ' $x$ ' is equivalent to whatever else, regardless, ' $x$ ' just is the case. 
- Existential certainty normally precludes the notion of uncertainty, and grounds the possibility of epistemic certainty and uncertainty. To become existentially uncertain/disturbed is not merely to lack confidence in some putative knowledge, it is for the structure of knowing and believing to begin falling apart. Rarely do we become uncertain in this way.

Point (1) is simply an allowable synonymity given connotational suppleness of the terms 'certain' and 'known'. I do not take this to be interesting in this context. Points (2) and (3) basically come together to form the epistemic confidence I have discussed in the 'Preparing students to be uncertain in an uncertain world: disentangling 'epistemic confidence' from 'certainty' section. By contrast, existential certainties - analysed as (4) of the above schemata-are expressions of those ways all cultures stabilise themselves as socio-historical forms of living, into which we are socialised as epistemic agents. Certainties constitute the unquestioned, stable framework against which we make sense of ourselves and the world around us. To be uncertain in this context is existentially devastating. As Wittgenstein puts it: 'when is something objectively certain? When a mistake is not possible...' (§194). The objectivity that he links to certainty is crucial because it indicates that the foundations of the certainty do not lie in the (knowing) subject, but in the socio-cultural milieu in which subjects make sense of themselves as subjects. Another way of putting this point is that we cannot question everything that we 'know' all at once. We have to hold some 'knowledge'(/ certainties) as a fixed ground upon which knowledge may be built. What we hold fixed in this way is not a result of personal choice-after all choice is in some relevant sense an epistemic activity - even if it seems as such. What we hold fixed as certain, are functions of large-scale socio-cultural forms of living which provide frameworks for the intelligibility of our social activities - including epistemic practices such as teaching and learning $(\S 410)$.

We can see here that the problematics of certainty explored here have resonances with issues highlighted by pedagogical theories of 'conceptual change' (CC). It is worth spending a moment on these resonances to emphasise the distinctiveness of the claim about existential certainty. CC arose from the likes of Kuhn's historical analysis of scientific practice. He argues that clashes of incommensurability between differing conceptual schemata present challenges for scientists that implicate entire paradigms within which they operate (Kuhn, 1962: 37). This theory has then inspired pedagogical analysis into student difficulty more generally: students experience learning difficulty when their pre-existing, conceptual understandings ('misconceptions') clash with theories of concepts as they are being taught (see Nussbaum and Novick 1982: 184; Posner et al. (1982) 212-3). It is hard for a student to learn some idea if an implication of what they already understand by a concept is at odds with, or cannot accommodate for, what is being taught. Furthermore, CC theorists have argued for augmenting the 'cold', somewhat cognitivist rendering of conceptual content and assimilation, with 'warm' parameters which involve student motivations and socio-cultural factors (see Strike and Posner (1992) 163; Pintrich, Marx and Boyle (1993) 182-5). What I say here clearly complements such theorising and has implications for it. However, CC is still first and foremost about epistemic clashes between conceptual schemata. Again, what is distinctive in my analysis is the consideration of existential dispositions that frame the epistemic domain. Warm CC, which considers socio-cultural factors that account for the acquisition and assimilation of conceptual schemata, still operates with a notion of concepts at the epistemic level. This is fine, and nothing I say is meant to undermine that analysis within the epistemic domain. Nevertheless, an existential certainty is a commitment that frames the intelligibility of such clashes. In other words, troubles that arise 
as a result of student certainties are not problems of misconception; breaking down those misconceptions through standard epistemic practices - such as demonstrating the failure in explanatory power of mistaken theories in the face of empirical evidence-are not apt for dealing with difficulties that arise out of existential certainties. Another way of putting the problem that teachers face in cases of existential certainty is that the material being taught will strike the student as unreasonable, and not merely false. In such a case why would we think that standard reasoning/epistemic practices could overcome the learning obstacle?

All that said, it may be thought, given the name example, that what count as certainties must be mundane or trivial. That is not the case. Certainties may be expressions of surprising richness and depth precisely because certainties are piecemeal articulations of the forms of living that congeal as cultures. Moreover, given that any culture has its certainties, as it were, it does not follow that the issues being explored here are particular to any culture. So, just because the European Enlightenment traditions that shape the cultural imaginary of 'Western' epistemology, which has historically tended to foreground the epistemic value of uncertainty/rational doubt, it does not follow that 'Western' cultures are 'above' having existential certainties. Nor is there anything in this view of existential certainty that privileges any particular cultural content: secular, scientific cultural forms of living have their certainties as much as any other. There is therefore, a strong degree of cultural relativism built into this theory. Nevertheless, even if that is a worry for the reader, the aim here is not to justify or argue for the view of certainty at hand, even though I would defend it. The point is to explore its explanatory power for TCF-justification of attendant commitments would have to be wrestled with after. Regardless, we find out how important, rich, and complex existential certainties are when we try to teach material which puts pressure on such foundations. I now want to consider such a scenario. This helps us to understand what might be happening with certainties, to elaborate the difficulties that arise for students and teachers alike, and how we might think of such certainty-involving conceptual material as candidates for a threshold concept. This example also demonstrates how a candidate threshold concept may turn out to be implicated in a socio-cultural certainty. As such, it is both relatively easy to identify by the distinctive existential trouble it causes for students, and to show that the troublesomeness engendered is far deeper than an individual's epistemic difficulty.

\section{When what we teach is 'certainly false': the epistemic limits and conditions for the possibility of teaching and learning}

I teach many areas of philosophy, from technical, analytic philosophy to poststructuralist gender theory. It is only in the latter domain that I have come across student difficulty that seems relevant to the kind of certainty discussed in the 'Existential certainty: epistemic frameworks and forms of living' section. When teaching Judith Butler's critique of the concepts 'sex' and 'gender', some students find the material distinctively troublesome. Indeed, student difficulty has often expressed itself in profoundly hostile and vehement forms: Butler's theory of 'sex' and 'gender' is 'clearly false', 'nonsense', 'stupid', 'unintelligible'. These kinds of responses appear to be in the vein of (4a) and (4b) above. It concerns a clash between discipline specific conceptual material and what students take to be certain; the phenomenological character of this difficulty is profound existential threat. In the case of Butler's theory of gender, we find a concept that ticks all the criteria of a threshold concept, is genuinely distinctive in its difficulty, and which can be theorised at as 
a function of socio-cultural states of affairs as opposed to merely 'subjective' difficulty. As such, poststructuralist analyses of 'sex' and 'gender' might render them exemplar threshold concepts given the analysis so far.

Firstly, we should note that Butler's analysis is a debate-setting position: any student engaging with poststructuralist analyses of 'sex' and 'gender' needs to understand her theory-and by that I do not suggest that they must agree with her. How then does this result in distinctive student difficulty? To answer this, some of her claims are useful. In her book Gender Trouble, Butler argues that the pre-theoretical/hegemonic/conservative view that 'sex' is metaphysically prior to 'gender', and determines gender, does not stand up to scrutiny - see Butler (2005: 10-22) for details of the argument. Moreover, the notion that there is some objective, biological state of affairs that stands apart from the normative entanglements of 'gender' is misguided. Finally, that our thinking about 'sex' is in fact coordinated by our thinking about 'gender', and not the other way around. No-one doubts, least of all Butler, that these are controversial claims that fundamentally challenge the ways that western cultures standardly think about 'sex' and 'gender'. Moreover, her view thereby challenges how many people understand their own subjectivity, identity, the social structures implicated in 'sex' and 'gender' (which are arguably all structures), and how science fits into the world.

What do students make of this idea about sex and gender? Unsurprisingly some like and are prepared to take it up, others wish to reject it. It is the latter group I am interested in. Students who reject Butler's position then fall into two camps: those who take the view to be appropriate for consideration, even if wrong in some way or other; and those who find the view literally nonsense. And it is this latter response that I think is crucial. In such cases, they are not saying that Butler is claiming something false; she is claiming something that it is unclaimable. In short, there are students for whom Butler is wrong, and there are students for whom Butler is certainly wrong. The way that I want to understand this is in terms of the epistemic analysis introduced in the 'Existential certainty: epistemic frameworks and forms of living' section. For some people, when Butler claims that 'sex' is a socio-cultural, historical concept, she may as well be saying she has eighteen heads! What is the situation where such a claim could even make sense let alone be right or wrong? It is one thing to say something that may be false, it is another to make a sentence that has only the semblance of meaning, i.e. syntactically well-formed, semantically sensical at the atomistic level, and yet when considered as a meaningful sentence becomes unintelligible.

One may think that students' epistemic responses of bafflement/hostility can be explained very simply: Butler is really wrong. Perhaps I should chalk up my teaching experiences to nothing more than teaching something daft; there are many voices who would think that the problem here is nothing to do with conceptual troublesomeness, but a theory that ought not to be taught at all. Here is not the place to argue for or against Butler's view, nor to justify her place on the curriculum. Regardless, student response to Butler as saying something certainly false is pedagogically important: it is a distinctive form of difficulty insofar that it is a use of a concept they cannot understand. Let us assume the problem is because Butler's view is just 'really out there'! But then there are other things on our philosophy curriculum that are considered mainstream, but which are far more 'out there' in particular respects. I also teach modal metaphysics which concerns the nature of 'possibility' - a core area in contemporary analytic philosophy. A significant view in this theory is that there are a countless infinity of alternate universes which really, concretely exist, that are knowable by thought alone, and which can help us make sense of a whole range of philosophically significant phenomena-see Lewis (1986: 133). It is a challenging position about possibility. But student difficulty, to use a modal notion, is not necessary 
to learning the concept. Indeed, some students' intellectual dispositions are such that they find it somewhat straightforward and compelling. Put another way, students may passionately argue about the nature of possibility and whether or not we should think about possibility in terms of 'possible worlds', but no student that I have ever met has found the theory of possible worlds beyond intelligibility. Yet, for Butler, the attempt to argue that 'sex' might be a function of 'gender' is, for some students, existentially repulsive.

I discuss these two examples, and their political import for teaching, in more detail elsewhere (Stopford, forthcoming). All that said, if we use the resources of part three, there is no mystery here. The claim 'sex is a natural phenomenon' is itself a socio-cultural and historical certainty - note that there are no scare quotes inside this proposition precisely because the concepts involved are not understood to be available for interpretation. Many students have been inaugurated into a socio-cultural and historical milieu that takes some assumed form of scientistic naturalism to be the grounds and possibility of epistemic intelligibility, especially on such matters as 'sex'. Furthermore, the socio-historical ways that cultures are organised as intelligible, coherent, communicative spaces within which students coordinate, and understand themselves, as epistemic agents, congeal as a fact-like framework within which epistemic activity can take place, and which leaves the framework of 'facts' beyond the scope of epistemic inquiry. Whatever 'sex' is, it is codified into the economic, political, and social structures that certain cultures consist in. Hence, some students are certain that Butler is wrong. It is worth noting, and it is implied in the above analysis, that difficulties arising from clashes between student certainties and discipline specific material will be more acute or preponderant in certain disciplines relative to student background. Again, this does not mean that some discipline will have intrinsically challenging material, but that difficulties could be anticipated with appropriate sensitivity to disciplinary content relative to constituencies of students.

\section{Conclusion}

The aim of this paper is to highlight a distinctive way that we are 'certain'. The most common way that the term gets used, I suggest, is to express a level of confidence in what we know. However, there is a more fundamental way that we are certain, which rarely gets expressed, precisely because it is fundamental. Such certainty requires excavation from its position as the bedrock of knowledge. It has not been the role of this paper to provide justification for such certainty either by formal epistemology nor Wittgenstein scholarship. It has been to introduce the idea and motivate it as we might find it in our teaching practices. Hopefully, the reader will find the idea prima facie plausible and that it will resonate with them. Moreover, to the extent that I might be right, such certainty is going to be crucial for us to negotiate in any kind of teaching practice, because we, and our students, all have existential commitments which we take to be certain. At the very least, if what we teach clashes with such certainties, student resistance will be much more profound than epistemic difficulty.

This view of certainty could have explicit usefulness for TCF. TCF is currently under theoretical pressure to provide a method for identifying TCs, and for convincing us that TCs are difficult in ways other than personal difficulty. Existential certainty could help TCF on both fronts. TCs would be that conceptual material which clashes with student certainty and so we could identify a TC by a student's reaction to it. Moreover, that reaction is not just a function of their own epistemic difficulty; it is due to their socio-cultural background 
which precludes the intelligibility of the TC. As I have suggested, adopting my account of certainty for explanatory power would require some changes to TCF. TCs are likely to be less preponderant than has been theorised hitherto. Secondly, they are not the difficult material of the discipline, they are difficult in a relational sense: as a clash between the socio-cultural background of the student and the concept as rendered in the discipline. I have not argued that TCF ought to adopt my view. However, if they want to reject my view of certainty and its explanatory benefits, TCF is back to the drawing board with no in principle way to establish that there even are TCs, let alone how to identify them, and a view of student difficulty that is no more than an important reminder that we have to attend to student difficulty — but no real method for how to deal with that difficulty.

Acknowledgements I would like to thanks Jana Cattien for her patient readings and re-readings of this paper. Thanks also to the peer reviewers for their time, effort and helpful commentary.

Funding University of Durham

Open Access This article is licensed under a Creative Commons Attribution 4.0 International License, which permits use, sharing, adaptation, distribution and reproduction in any medium or format, as long as you give appropriate credit to the original author(s) and the source, provide a link to the Creative Commons licence, and indicate if changes were made. The images or other third party material in this article are included in the article's Creative Commons licence, unless indicated otherwise in a credit line to the material. If material is not included in the article's Creative Commons licence and your intended use is not permitted by statutory regulation or exceeds the permitted use, you will need to obtain permission directly from the copyright holder. To view a copy of this licence, visit http://creativecommons.org/licenses/by/4.0/.

\section{References}

Barradell, S. (2013). The Identification of threshold concepts: a review of theoretical complexities and methodological challenges. Higher Education, 65, 265-276.

Bleakley, A, \& Cleland, J. (2015) Sticking with messy realities: 'how thinking with complexity' can inform healthcare educational research. In Researching Medical Education. Eds. J Cleland and S. Durning. Wiley: Oxford.

Booth, J. (2006) On the mastery of philosophical concepts: Socratic discourse and the unexpected 'affect'. In Overcoming Barriers to Student Undertanding. Eds. J. Meyer \& R. Land. Routledge: London.

Butler, J. (2005). Gender Trouble. London: Routledge.

Cartensen, A., \& Bernhard, J. (2008). Threshold concepts and keys to the portal of understanding. In R. Land, J. H. F. Meyer, \& J. Smith (Eds.), Threshold Concepts Within the Disciplines (pp. 143-154). Rotterdam: Sense Publishers.

Foley, B. (2011). Threshold concepts in disciplinary settings. Cork, Ireland: Paper presented at the Threshold Concepts Symposium.

Hill, S. (2020). The difference between troublesome knowledge and threshold concepts. Studies in Higher Education., 45, 3 .

Kuhn, T. (1962). The structure of scientific revolutions. Chicago: Chicago University Press.

Land, R. (2011). Threshold concepts and troublesome knowledge. Cork, Ireland: Paper presented at the Threshold Concepts Symposium.

Land, R. (2016). Toil and trouble: Threshold concepts as a pedagogy of uncertainty. In R. Land, J. H. F. Meyer, \& M. Flanagan (Eds.), Threshold concepts in practice (pp. 11-24). Rotterdam/Boston/Taipei: Sense Publishers.

Lewis, D. (1986). On the plurality of worlds. Oxford: Basil Blackwell.

Lloyd, P., \& Frith, V. (2013). Proportional reasoning as a threshold to numeracy at university: A framework for analysis. In Pythagoras, 34(2), Art. \#234.

Male, S., \& Baillie, C. (2011). Threshold capabilities: an emerging methodology to locate curricula thresholds. Madrid, Spain: Paper presented at Research in Engineering Education Symposium.

Meyer, R., \& Land, J. (2003). Threshold concepts and troublesome knowledge: Linkages to ways of thinking and practicing within the disciplines. ETL Project, Occasional Report, 4, 1-12. 
Meyer, R., \& Land, J. (2005) Threshold concepts and troublesome knowledge (2): Epistemological considerations and conceptual framework for teaching and learning. In Higher Education, 49, 373-388.

Meyer, J., Land, R., \& Davies, P. (2008). Threshold concepts and troublesome knowledge (4): Issues of variation and variability. In R. Land, J. Meyer, \& J. Smith (Eds.), Threshold concepts within the disciplines (pp. 59-74). Sense: Rotterdam.

Nicola-Richmond, K., Pépin, G., Larkin, H., \& Taylor, C. (2018). Threshold concepts in higher education: A synthesis of the literature relating to measurement of threshold crossing. Higher Education Research \& Development, 37(1), 101-114.

Nussbaum, J., \& Novick, S. (1982). Alternative frameworks, conceptual conflict and accommodation: Towards a principled teaching strategy. Instructional Science, 11(3), 183-200.

Quinlan, K. M., Male, S., Baillie, C., Stamboulis, A., Fill, J., \& Zaffer, Z. (2013). Methodological challenges in researching threshold concepts: A comparative analysis of three projects. Higher Education, $66,585-601$.

O'Donnell, R. (2010) A critique of the threshold concept hypothesis and an application in economics. Working Paper No. 164.

Pintrich, P., Marx, R., \& Boyle, R. (1993). Beyond cold conceptual change: The role of motivational beliefs and classroom contextual factors in the process of conceptual change. Review of Educational Research, 63(2), 167-199.

Posner, G., Strike, K., Hewson, P., \& Gertzog, W. (1982). Accommodation of a scientific conception: Toward a theory of conceptual change. Science Education, 66(2), 211-227.

Rattray, J. (2016). Affective dimensions in liminality. In R. Land, J. H. F. Meyer, \& M. Flanagan (Eds.), Threshold concepts in practice (pp. 67-76). Rotterdam/Boston/Taipei: Sense Publishers.

Rowbottom, D. (2007). Demystifying threshold concepts. Journal of Philosophy of Education, 41(2), $263-270$.

Salwén, H. (2019) Threshold concepts, obstacles or scientific dead ends? In Teaching in Higher Education. $1-14$.

Shanahan, M. (2016). Theshold concepts in economics. Education and Training., 58(5), 510-520.

Shulman, L. (2005). Pedagogies of uncertainty. In Liberal Education(Spring issue).

Stopford, R. (forthcoming) Teaching feminism: Problem of critical claims and student certainty. In Philosophy and Social Criticism.

Strike and Posner (1992) A Revisionist Theory of Conceptual Change. In Philosophy of Science, Cognitive Psychology, and Educational Theory and Practice. Eds. R. Duschl and R. Hamilton. State University of New York Press: New York.

Taylor, C. (2006). Threshold concepts in biology do they fit the definition? In J. H. F. Meyer \& R. Land (Eds.), Overcoming Barriers to Student Understanding Threshold Concepts and Troublesome Knowledge (pp. 87-99). London: Routledge.

Taylor, C. (2008). Threshold concepts, troublesome knowledge and ways of thinking and practicing. In R. Land, J. H. F. Meyer, \& J. Smith (Eds.), Threshold Concepts Within the Disciplines (pp. 185-195). Rotterdam: Sense Publishers.

Timmermans, J. \& Meyer, J. (2019) Embedding affect in the threshold concepts framework. In Threshold Concepts on the Edge. Eds. J. Timmermans \& J. Meyer. Educational Futures, Vol. 73. Sense: Rotterdam.

White, B., Olsen, T., and Schmann, D. (2016) A threshold concept framework for use across disciplines. In Threshold Concepts in Practice. Eds. R. Land, J. H. F. Meyer and M. Flanagan. Sense Publishers: Rotterdam/Boston/Taipei.

Wittgenstein, L. (1972) On certainty. Trans. G. E. M. Anscombe \& G.H. von Wright. Blackwell: Oxford.

Publisher's Note Springer Nature remains neutral with regard to jurisdictional claims in published maps and institutional affiliations. 